\title{
A New Direction for Closed-Loop Spinal Cord Stimulation: Combining Contemporary Therapy Paradigms with Evoked Compound Action Potential Sensing
}

\author{
Ricardo Vallejo' \\ Krishnan Chakravarthy ${ }^{2}$ \\ Andrew Will ${ }^{3}$ \\ Karen Trutnau ${ }^{3}$ \\ David Dinsmoor (iD) ${ }^{4}$ \\ 'Millennium Pain Center, Bloomington, IL, \\ USA; ${ }^{2}$ Anesthesiology and Pain \\ Management, University of California San \\ Diego, San Diego, CA, USA; ${ }^{3}$ Twin Cities \\ Pain Clinic, Edina, MN, USA; \\ ${ }^{4}$ Neuromodulation Research \& Technology, \\ Medtronic plc, Minneapolis, MN, USA
}

\begin{abstract}
Spinal cord stimulation (SCS) utilizes the delivery of mild electrical pulses via epidural electrodes placed on the dorsal side of the spinal cord, typically to treat chronic pain. The first clinical use of SCS involved the delivery of paresthesia inducing, low-frequency waveforms to the neural targets corresponding to the painful areas. Contemporary SCS therapies now leverage novel therapeutic pathways to limit paresthesia and deliver superior clinical outcomes. Historically, SCS has largely been delivered with fixed stimulation parameters. This approach, referred to as open-loop (OL) SCS, does not account for the fluctuations in spacing - driven by postural changes and activity — between the electrodes and the cord. These fluctuations result in variability in the delivered dose and the volume of tissue activation (VTA) that manifests with each stimulation pulse. Inconsistent dosing may lead to suboptimal therapeutic efficacy and durability. To address this clinical need, closed-loop (CL) SCS systems have been developed to automatically adjust stimulation parameters to compensate for this variability. The evoked compound action potential (ECAP), a biopotential generated by the synchronous activation of dorsal column fibers, is indicative of the VTA resulting from the stimulation pulse. The ECAP may be utilized as a control signal in CL SCS systems to adjust stimulation parameters to reduce variability in the ECAP, and in turn, variability in the VTA. While investigational CL SCS systems with ECAP sensing have so far focused solely on managing paresthesia-based SCS, such systems must also incorporate the stimulation approaches that now define the contemporary clinical practice of SCS. Accordingly, we describe here a flexible, next-generation framework for neural responsive SCS that blends science-based methodologies for pain management with real-time CL control for biophysical variation. We conclude with a clinical example of such a system and the associated performance characteristics.
\end{abstract}

Keywords: spinal cord stimulation, evoked potentials, closed-loop, neuromodulation

\section{Spinal Cord Stimulation: Established Intervention and Emerging Innovations}

Development of spinal cord stimulation (SCS) as a pain therapy over the last halfcentury has been characterized by dramatic innovative shifts. In its earliest iterations, SCS used one to four epidural electrodes ${ }^{1-3}$ and the implanted technology was powered by a bulky, externally worn radiofrequency controller. ${ }^{4}$ In the early 1980 s, pulse generators became fully implantable with wireless handheld controllers. ${ }^{5}$ Improvements
Correspondence: David Dinsmoor Neuromodulation Research \& Technology, Medtronic plc, 7000 Central Ave NE RCE275, Minneapolis, MN, 55432, USA $\mathrm{Tel}+\mid 763$ 526-880 I

Email david.a.dinsmoor@medtronic.com 
such as rechargeable battery technology and computercontrolled programming soon followed, ${ }^{6,7}$ providing patients with previously impossible options for using high-energy stimulation programs and creating nuanced paresthesia coverage. As compared to open loop (OL) systems where the patient or clinician is responsible for all parameter changes, closed loop (CL) systems were introduced that automatically controlled stimulator settings based on sensor inputs. ${ }^{8,9}$ User acceptability grew and SCS established a position well within the mainstream options for chronic pain management.

From these foundations, SCS manufacturers expanded their treatment portfolios to include new waveforms, neurological targets, and device sizes and shapes. ${ }^{10-14}$ Knowledge about SCS mechanisms of action have now extended far beyond the traditional gate control theory ${ }^{15}$ to include an appreciation for incredibly complex control involving molecular systems, cell types, metabolic/systemic processes, and neural pathway activation (eg, $\left.{ }^{15-21}\right)$. Contemporary therapy paradigms leverage a mechanistic understanding of these pathways and deliver multiple stimulation modalities to optimize clinical outcomes (eg, the concurrent application of multiple stimulation parameters within programs characterizing differential target multiplexed (DTM) SCS (Medtronic plc), depicted in Figure 1). ${ }^{22-24}$

As such, SCS is now situated at the confluence of both the technical and clinical advancements that have defined the field, particularly in the last decade. In this report, we provide perspective on a new direction for spinal cord stimulation that unites the latest therapeutic paradigms with one of the newer SCS technologies available - CL control using the spinal evoked compound action potential (ECAP) as a feedback control variable. We discuss exemplary data and potential performance measures for such systems and describe future opportunities as this field continues to evolve and expand.

\section{The Evoked Compound Action Potential}

ECAPs are the electrophysiological recordings of the collective voltage change in the extracellular matrix surrounding bundles of axons that are simultaneously activated by an electrical pulse delivered by an electrode. The recording electrodes used to detect ECAPs are typically stationary and located some distance from the stimulating electrodes; they detect the axons' action potentials as they proceed

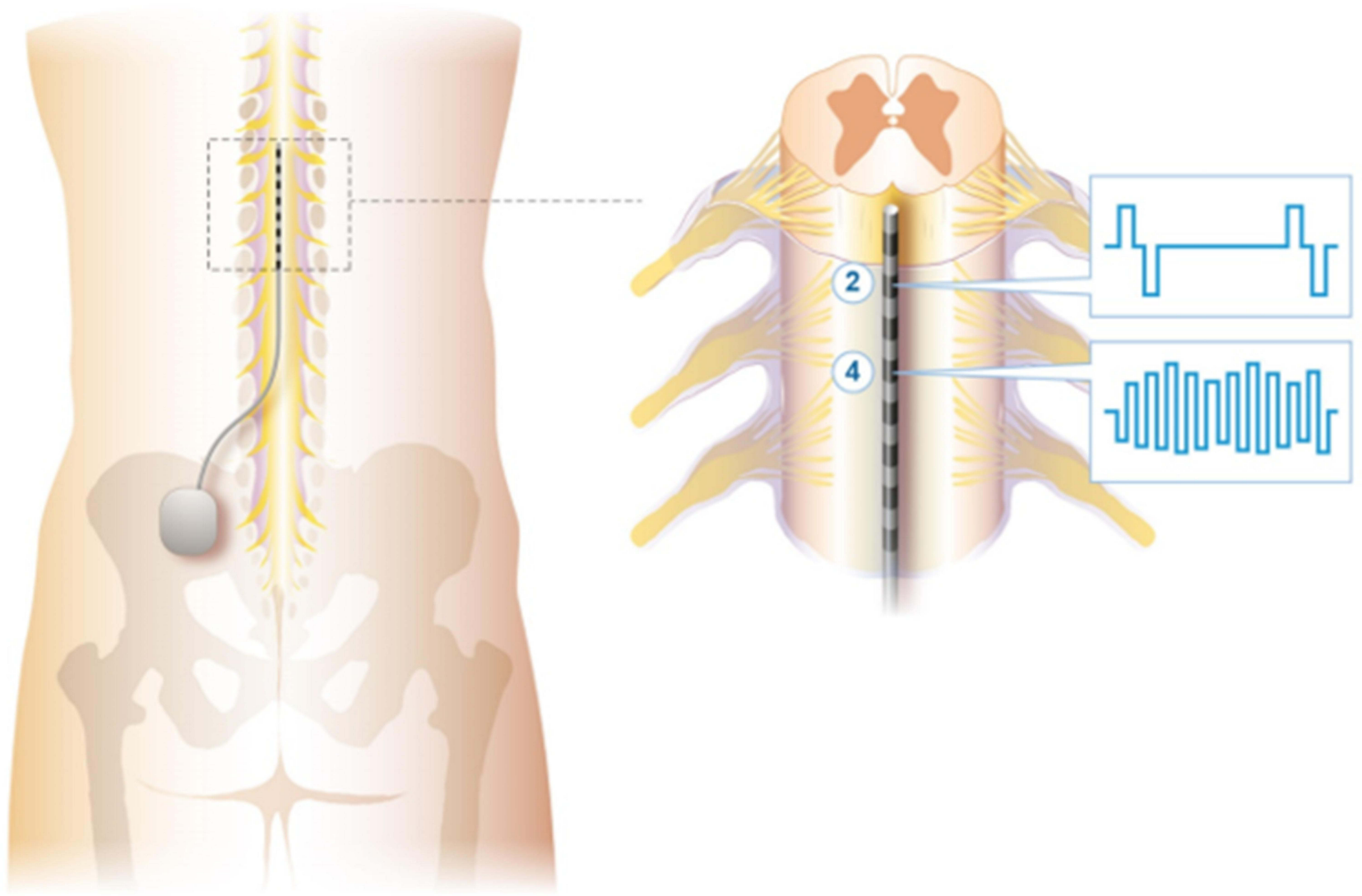

Figure I An implanted spinal cord stimulation system with a lead positioned in the epidural space is used to deliver multiplexed stimulation patterns (a fixed amplitude, low frequency program and a variable amplitude, high frequency program) to differential targets (electrodes 2 and 4 ) on the dorsal columns of the spinal cord. 
either orthodromically or antidromically from the site of stimulation. Clinically, ECAP recordings have been made, among other locations, at muscles, ${ }^{25}$ peripheral nerves, ${ }^{26}$ cochlear nerves, ${ }^{27}$ and the spinal cord. ${ }^{28}$

Displayed on an oscilloscope, the spinal ECAP has a typically triphasic shape, with an initial positive voltage peak (termed P1, occasionally observed) followed by a negative deflection (N1) and a second positive peak (P2) (Figure 2), and are strongly influenced by the contribution of large-diameter fibers. ${ }^{29}$ The amplitude of the ECAP - defined here as the differential voltage between the $\mathrm{N} 1$ and P2 features of the ECAP - is indicative of the presumed spinal volume of tissue activation (VTA) elicited with the stimulation. ${ }^{29,30}$ Although a volumetric measure, VTA is often conceptualized in cross-section. The VTA in the dorsal columns contains ascending dorsal column fibers. A small VTA in the dorsal columns of the spinal cord would presumably be indicated by a small number of

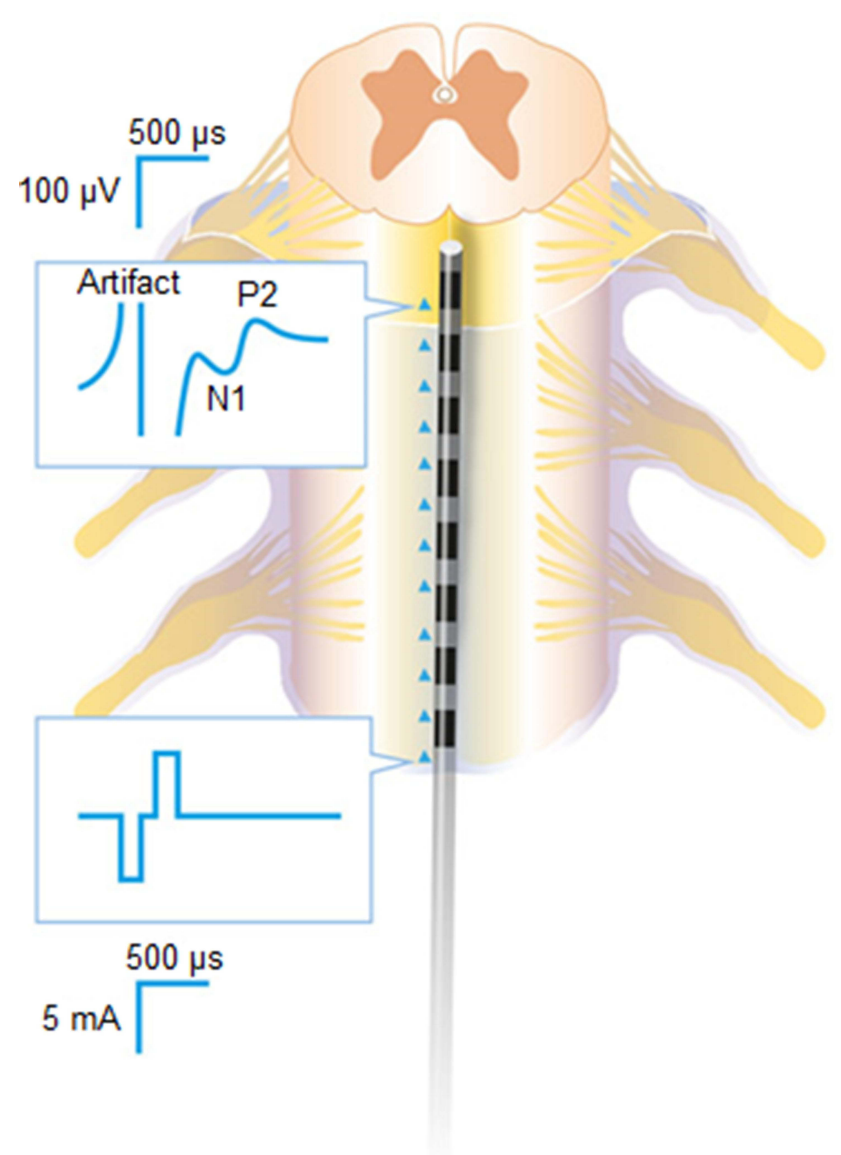

Figure 2 Segment of spinal cord with stimulation applied to the dorsal columns one end of a lead, with the resultant evoked compound action potential (ECAP) and associated artifact measured on the opposite end. $\mathrm{NI}$ and P2 refer to the first trough and second peak, respectively, of the ECAP. individual axons that are recruited to contribute to the small ECAP; conversely, a large VTA would be represented by a larger number of individual axons and a larger ECAP. While a large VTA elicited with dorsal column stimulation may directly result in nociceptive phenomena such as lateral root stimulation, it is important to remember that the ECAP itself is not a biomarker of pain.

The recording electrodes also detect stimulation artifacts, observed as high-amplitude spikes caused by the stimulation pulses themselves. Stimulation artifacts occur at the same time as the onset of the ECAP but are only noise - not part of the ECAP itself. Depending on its magnitude (amplitude and duration), the artifact may obscure the relatively subtle ECAP signal. The effect of the artifact can be lessened by ensuring sufficient spacing between the stimulating and recording electrodes, so that — based on the conduction velocity of the recruited fibers - the ECAP arrives later than the artifact. Another way to limit contamination of the ECAP signal by stimulation artifact is via the use of relatively shortpulse widths that result in narrow artifacts. ${ }^{30}$

Additionally, stimulation artifact suppression schemessuch as high-pass filtering and template correlation approaches - have been developed to limit the extent of artifact contamination on the ECAP estimate. This is a critically important step toward being able to interpret ECAPs and use them for neurophysiological applications. If artifact processing is inadequate, it could lead to the artifact being erroneously classified as "true" neural response. This may have clinically meaningful consequences for the follow-on functions that depend on accurate ECAP measurement, such as CL SCS systems that use the ECAP as a feedback control variable to inform stimulation adjustments. $^{31}$

\section{Compensating for Motion: Closed-Loop Control Considerations for SCS}

The clinical need for CL SCS is motivated by variability in the spacing between the spinal cord and the epidurally located SCS leads based on the patient's position or movement. For example, this spacing changes by $2.2 \mathrm{~mm}$ on average for supine vs prone positioning due to the effects of gravity on the spinal cord. ${ }^{32}$ Changes in posture for a person with an indwelling SCS system therefore result in changes in the effective distance between the leads and the target tissue. Assuming that the amplitude of stimulation is held constant, this results in a larger or smaller VTA in the dorsal spinal cord. All SCS therapies-regardless of the 
specific therapeutic approach employed - are susceptible to this variability in VTA with lead motion. In conjunction with selecting the optimal dose, consistent dosing - that is, maintaining a consistent VTA throughout therapy-is critical to optimizing therapeutic outcomes. ${ }^{33}$

The complexity in treating ambulatory patients with SCS is illustrated by the fact that the amount of energy required to achieve comfortable SCS treatment while standing, sitting down, lying down, and walking is markedly different. ${ }^{34,35}$ Other movements, such as bending at the waist, or stretching the limbs, may likewise initiate such changes in lead vs spinal cord positioning. Because changes in stimulation intensity can be uncomfortable, patients manually adjust the amplitude of their devices. This has long been a complaint for the SCS intervention.

Posture-responsive stimulation was the first robust technology to address this problem. An on-board accelerometer detected the patient's position or movement and used CL feedback to automatically increase or decrease the amplitude of stimulation accordingly. When used in permanently implanted subjects in a 6-week crossover trial, subjects reported that PRS trended toward better pain relief than conventional SCS (in which all changes in amplitude were manual). With posture-responsive stimulation, subjects manually adjusted their stimulation an average of 18.2 times per day in addition to the automatic adjustments, which was $41 \%$ fewer button-presses than when using conventional SCS. ${ }^{9}$ Similarly, better pain relief and higher satisfaction for posture-responsive stimulation compared to conventional SCS have been reported in large retrospective comparative trials after 3 months $(n=96)^{36}$ and 24 months $(n=187) .{ }^{37}$

Accelerometer-based CL feedback is a robust tool for automatically adjusting stimulation to compensate for changes in the spacing between SCS leads and the spinal cord based on body positions. In some instances, changes in this spacing may not be solely attributable to postural variation; for example, cord motion may occur with coughs/sneezes or in response to subtle but ongoing movements like heartbeat or breathing. Posture-responsive stimulation, then, may be insensitive to these factors. A more nuanced option may be needed.

\section{The Spinal ECAP as a Closed-Loop, Feedback Control Variable}

Recognizing the clinical benefits realized with postureresponsive stimulation, quantitative sensors for SCS have been extended from accelerometers in the implanted stimulator to allow CL control via electrical measurement from the spinal cord itself. ${ }^{38,39}$ Here, spinal ECAPs are measured with every stimulation pulse and subsequently processed by a sophisticated algorithm in the implanted stimulator. If stimulation is delivered at $50 \mathrm{~Hz}$, for instance, ECAPs are measured every $20 \mathrm{msec}$; each measured ECAP provides quantitative insight into the degree and type of coupling between the stimulation signal and the spinal cord. SCS systems can leverage the rapid, repeated assessment of neural activation afforded by the ECAP as part of a highly responsive control system. CL SCS systems incorporating ECAPs as a feedback control variable offer a potential benefit over posture-responsive stimulation alone for conditions that result in an abrupt change in electrode-tissue spacing but no associated change in posture (such as a sneeze).

The interdependencies between lead motion, stimulation amplitude, the ECAP, and the VTA for both OL and CL SCS while a patient coughs are illustrated in Figure 3. As shown in this figure, the change in intrathoracic pressure resulting from the cough leads to a transient decrease in the spacing between the lead and the spinal cord. For the OL case (Figure 3A), the decreased spacing results in two effects. The first is an increase in the VTA, and the second is a spike in the ECAP amplitude as more signal generating fibers are activated by the stimulation. Patients may perceive this effect as an uncomfortable surge in stimulation intensity, or otherwise suboptimal SCS.

For the CL case (Figure 3B), the growing ECAP is sensed by the stimulator and interpreted as a control signal to decrease the stimulation amplitude. The decreased stimulation amplitude compensates for the decreased spacing between the lead and the spinal cord, and results in a controlled VTA and ECAP amplitude. For the patient, this may be perceived as an even perception of stimulation (if applicable) with optimized delivery of the SCS dose.

\section{Moving Beyond Controlled Paresthesia}

The approach employed by some investigational CL SCS systems involves adapting the stimulation amplitude of a patient's single, low-frequency program to maintain the ECAP within a paresthesia window. ${ }^{38}$ The lower bound of this window is the ECAP amplitude associated with the lowest stimulation amplitude that elicits perceptible paresthesia. The upper limit is the ECAP amplitude associated 


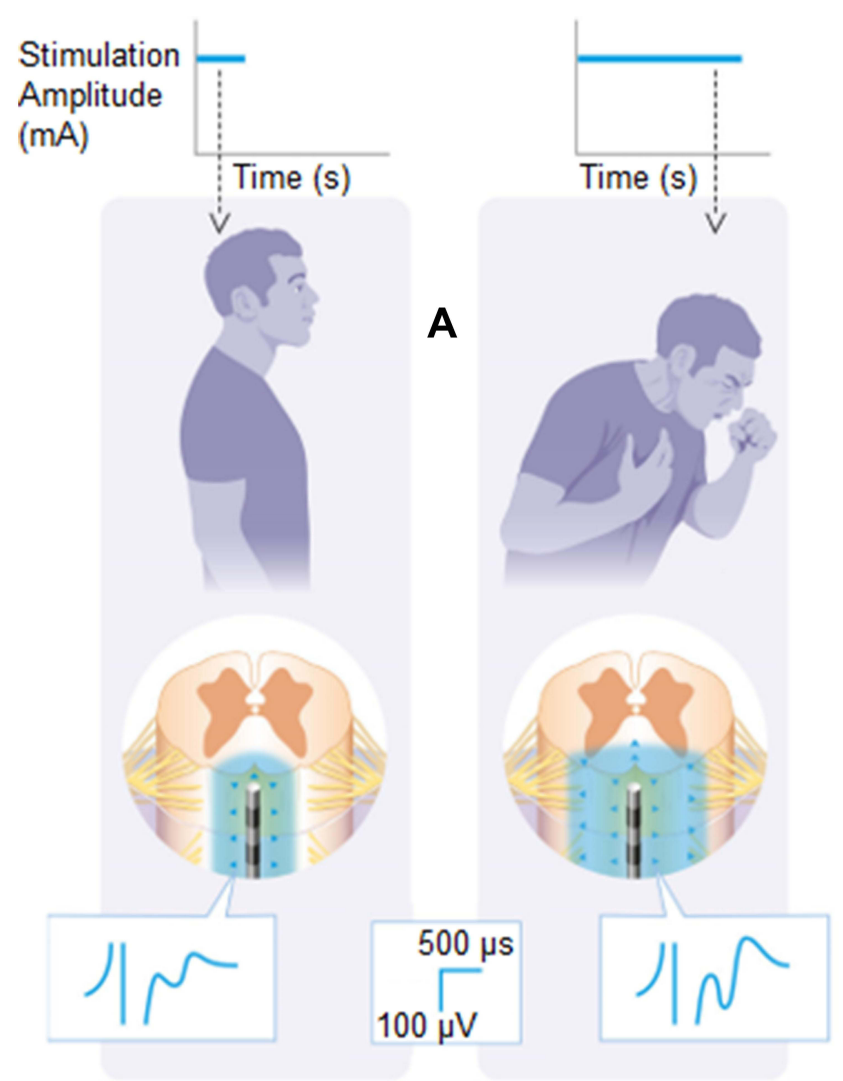

Open Loop SCS

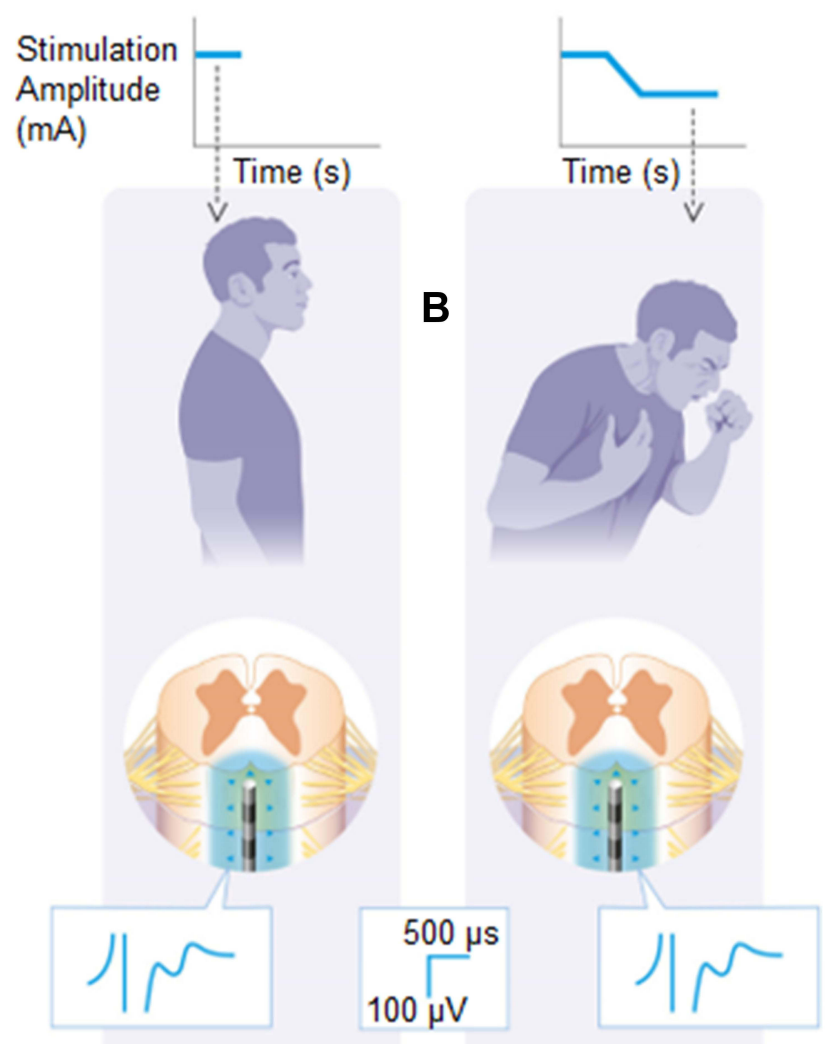

Closed Loop SCS

Figure 3 When the patient coughs, the spinal cord stimulation leads move closer to the spinal cord. This is detected by the spinal sensors as an increase in evoked compound action potential (ECAP) amplitude. In the open-loop configuration (A), the stimulation parameters are fixed; as such, the ECAP and volume of tissue activated (VTA, the blue area surrounding the electrodes) grows with the electrode-cord spacing decrease. In the closed-loop configuration (B), the system senses the increasing ECAP and automatically decreases the stimulation amplitude to maintain a constant VTA and ECAP amplitude.

with the highest stimulation the patient can maximally tolerate. This intervention necessitates the use of an SCS waveform that always incorporates perceptible paresthesia. Mechanistically, the neural circuits implicated with this approach are those of the well-known gate theory of pain, detailed over a half-century ago. ${ }^{15}$ As discussed in the Spinal Cord Stimulation: Established Intervention and Emerging Innovations section, however, the science of neuromodulation for pain control has advanced beyond the gate theory that simply provides paresthesia-heavy stimulation for the patient. In order to provide sophisticated CL SCS that is also clinically relevant, the ECAP as a feedback control variable must also be incorporated with the contemporary stimulation paradigms that are now foundational to the practice of SCS.

A framework that supports concurrent sensing of ECAPs and associated closed-loop control with modern neuromodulation paradigms is shown in Figure 4. This figure illustrates a next-generation approach to CL SCS using ECAPs, in which multiple signals comprised different parameters or frequencies are delivered in a timeinterleaved manner at different electrodes on the same lead. Potentially, more than two programs could be used.

Program \#1 in Figure 4 is a low-frequency signal delivered by stimulating electrodes at one end of the lead. The ECAPs elicited by Program \#1 are sensed by recording electrodes at the other end of the lead. This program is used as a "range-finder" to continuously assess the extent and type of neural activation via the ECAP. Additionally, Program \#1 may also serve as an important component of the patient's overall SCS therapy. Characteristics of the ECAP (such as the ECAP amplitude) are compared versus a target at every pulse. The difference between the ECAP and the target are used as part of a feedback loop to adjust subsequent stimulation pulses (Figure 4A). Changes can be made to a stimulation parameter (such as amplitude or frequency) to approximate a consistent VTA or consistent experience of therapy. In 


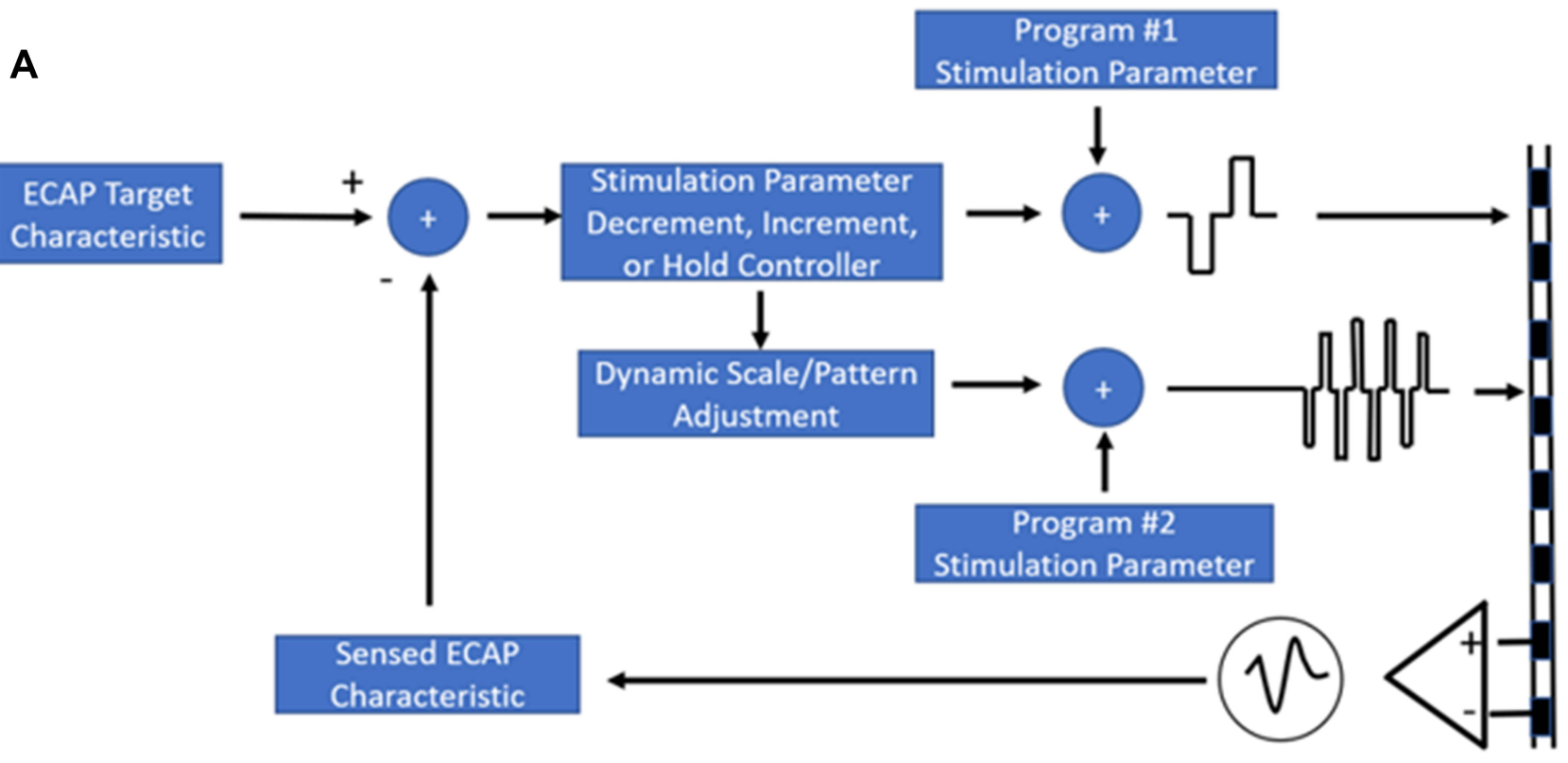

B

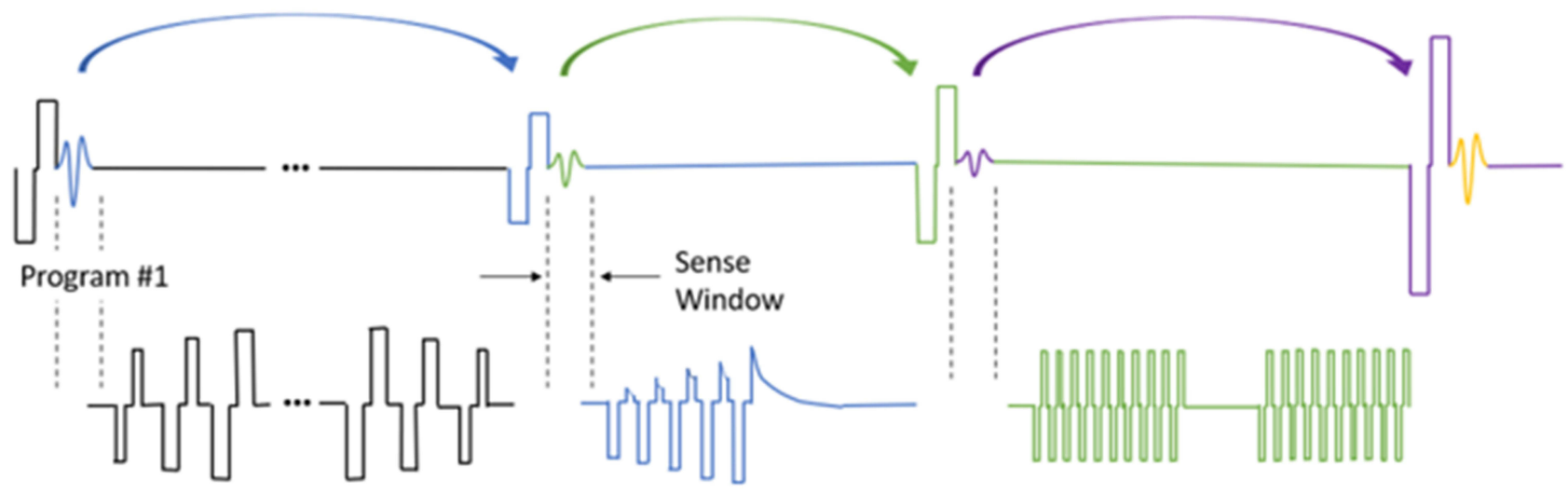

Program \#2

Figure 4 Evoked compound action potential (ECAP) sensing in response to each pulse from Program \#I allows for closed-loop control of both Program \#I and \#2, as governed by the feedback loop in (A). Multiple "therapy frames" (stimulation patterns with a common color) are shown in (B). The ECAP elicited from the pulse in Program \#I for one therapy frame informs the characteristics of the subsequent frame; this framework enables frame-by-frame volume of tissue activation control with multiple waveform patterns.

some instances, no ECAP may be the desired state, such as when sub-perception stimulation is the therapeutic intent. ${ }^{40}$ In that instance, the system can be configured to reduce stimulation amplitudes as soon as ECAPs corresponding to the patient's perceptual threshold are detected. Controlling stimulation in this manner lends itself to a system that does not rely on the continuous presence of ECAPs with associated paresthesia but rather on the prevention of them to optimize the target subthreshold stimulation parameters.

Program \#2 in Figure 4 is delivered by different electrodes than that used for Program \#1 or used for recording ECAPs. Program \#2 may also use different waveforms than Program \#1. The stimuli of Program \#2 are timeinterleaved between the pulses of Program \#1. The intent of Program \#2 is to effect outcomes that are difficult or not possible with Program \#1 alone, such as modulation of pain relevant biochemical pathways, ${ }^{17}$ neural inhibition with high-frequency stimulation, ${ }^{41}$ or bilateral delivery of the SCS. ${ }^{42}$

The neural response to Program \#2 is not sensed; this is because the characteristics of Program \#2 may be unsuited for eliciting an ECAP or recording an ECAP unencumbered by artifact. This is particularly true when high SCS frequencies, low amplitudes, or long pulse widths are used as applicable for optimal pain relief. Even so, Program \#2 
parameters can still be under CL control by simply yoking them to the same ECAP-based feedback loop that adjusts Program \#1. This way, where there is a detectable change in Program \#1's ECAPs, adjustments can be made in amplitude, frequency, or pulse width of both Program \#1 and Program \#2. Adjustments can be scaled in a ratiometric fashion, depending on the relationship between the two programs. The combination of stimuli comprising Program \#2 and preceding stimulus from Program \#1 are referred to as a "therapy frame."

As an example, we consider a Program \#1 that consists of a $40 \mathrm{~Hz}, 200 \mu$ s pulse width, $4 \mathrm{~mA}$ waveform delivered to electrodes 0 and 1 (E0/E1). The ECAPs elicited from this waveform are measured antidromically on E6/E7. Concurrent Program \#2 consists of a cluster of 5 pulses at $500 \mathrm{~Hz}, 1 \mathrm{msec}$ wide, and $2 \mathrm{~mA}$ in amplitude, delivered on $\mathrm{E} 3 / \mathrm{E} 4$. This cluster, which is interleaved between the stimulation pulses of Program \#1, is delivered outside of the "sense window" in Figure 4 to not disrupt the ability to resolve the ECAPs elicited with Program \#1. If the patient coughs, the system would detect that the ECAPs elicited with Program \#1 get larger. This means that the VTA is increasing because of a decrease in the electrode-tissue spacing. Without adjustment, the patient would experience this as a surge in stimulation intensity or otherwise sub-optimal therapy. Instead, the CL SCS compensates by reducing stimulation intensity. Because the stimulation amplitude ratio between Program \#1 and Program \#2 is 2:1 (4 mA vs $2 \mathrm{~mA}$ ), Program \#1 is decreased by $1 \mathrm{~mA}$ and Program $\# 2$ is decreased by $0.5 \mathrm{~mA}$. If needed, adjustments can be made as frequently as pulses occur in Program \#1, because the characteristics of the resultant ECAP informs the parameters for the next therapy frame. In concept, this approach could be applied to therapy frames that use multiple types of waveforms (eg, envelope-modulated, clustered, high-frequency), or that periodically switch between them.

Accelerometer- and ECAP-based CL control of SCS systems can be complementary and may be amenable to simultaneous deployment in the same device. As mentioned, ECAP sensing may provide nuanced or high-resolution control above and beyond that possible with accelerometer-based systems. Conversely, in some instances, it may not be possible to fully use ECAP control due to anatomy (eg, spinal canal size, CSF thickness) or lead placement. In these cases, accelerometerbased control would still be effective. Moreover, the two may work additively, for instance by scaling the ECAP target based on body position. ${ }^{43}$ Thus, the two approaches are not mutually exclusive but instead may readily integrate.

\section{In the Clinic: Closed-Loop, Differential Target, Multiplexed SCS}

Using CL DTM-SCS as a technical exemplar, the performance of the approach described above was evaluated in the clinic as part of the ECHO-MDT study. ${ }^{44}$ This study was a nonsignificant risk feasibility trial assessing the effects of stimulation parameters, electrode choice, activity, and processing methods on CL SCS control. All human clinical work for this multi-site, US-based study was approved by Western Institutional Review Board (WIRB \#20192532) and was conducted in accordance with the Declaration of Helsinki. We obtained written informed consent from each subject.

In this study, we tested ambulatory human subjects with a custom, investigational research system capable of delivering SCS, recording ECAPs, and adapting stimulation parameters on a frame-by-frame basis. The subjects were already undergoing commercial SCS trialing according to approved labelling; at the end of the commercial trial and prior to trial lead removal, we connected the subjects' 8-contact percutaneous leads (model 977D260, Medtronic plc) to the research system. For each subject, we personalized the research system's control parameters and stimulation intensity (charge per phase, or Q/ ph) of Program \#1 (Figure 4A) to optimize comfort and mitigate variability in the presumptive VTA over a range of common motions of daily living, such as back arches, torso twists, and coughs. Here, the role of Program \#1 is two-fold - both as the $50 \mathrm{~Hz}$, "base" therapeutic component of DTM, as well as the SCS signal that elicits an ECAP. The stimulation intensity of Program \#2 (Figure 4A), which serves as the higherfrequency multiplexed "priming" component of DTM, was set relative to Program \#1. ECAPs were then measured while subjects performed motions of daily living while the research system was operated in both OL and CL modes.

In an exemplary subject, we delivered Program \#1 at a nominal intensity of $0.54 \mu \mathrm{C} / \mathrm{ph}$ to electrodes 0 and 1 . Program \#2 was delivered at a nominal amplitude of $0.15 \mu \mathrm{C} / \mathrm{ph}$ to electrodes 2 and 4 (a ratio of 3.6:1). The ECAP elicited with Program \#1 was sensed on electrodes 6 and 7; this measurement occurred every $20 \mathrm{msec}$ following each pulse from Program \#1. The CL controller was configured to rapidly reduce stimulation intensity ratiometrically for both programs when the sensed ECAP amplitude exceeds $20 \mu \mathrm{V}$, a value approximating the subject's perceptual threshold. The system gradually raises stimulation intensity up to their nominal settings when the measured ECAP is below this threshold.

Depicted in Figure 5 is the CL DTM-SCS system performance; here, the subject is asked to perform motions of 

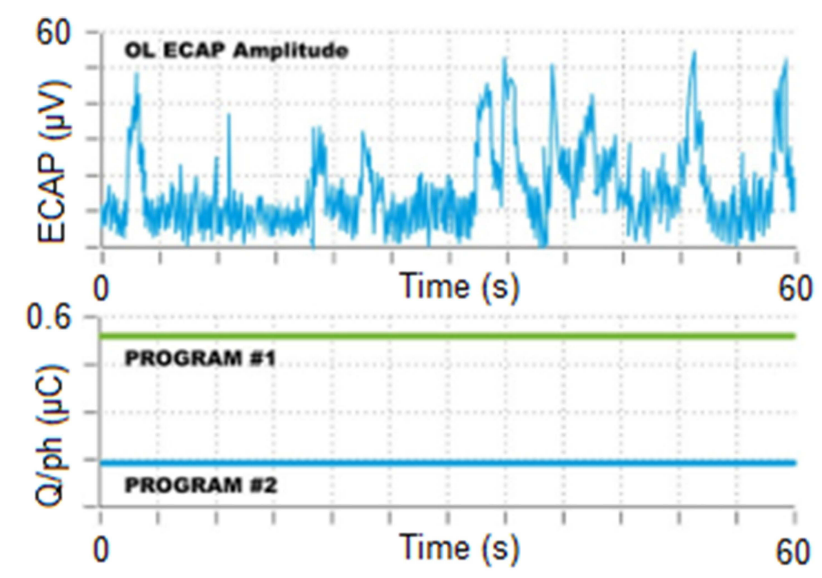

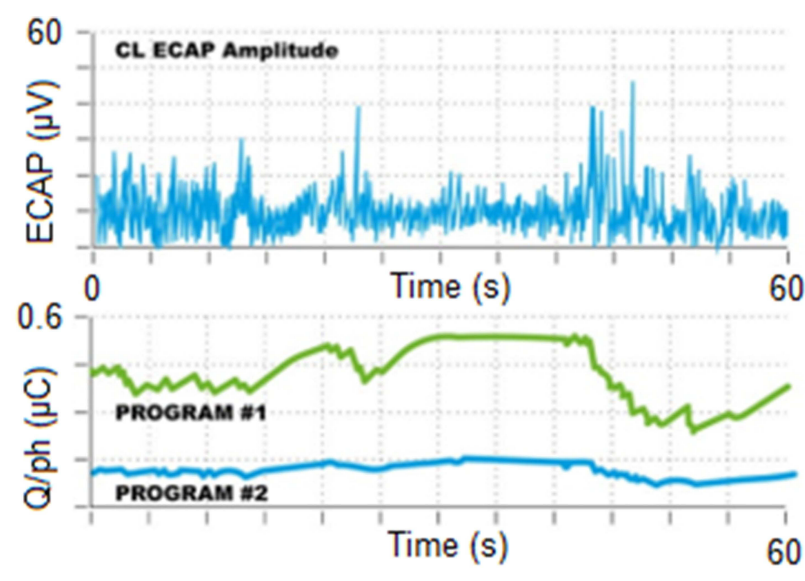

Figure 5 Evoked compound action potential (ECAP) amplitudes (top row) and multi-program charge/phase (bottom row) for both open-loop (OL, left) and closed-loop (CL, right) stimulation in a representative subject. Stimulation charge/phase, or $\mathrm{Q} / \mathrm{ph}$ (expressed as micro-coulombs/stimulation phase) is the product of stimulation amplitude (in milliamps) and stimulation pulse width (in milliseconds). The subject is asked to perform motions of daily living in both the OL and CL configurations, with the resultant variability in ECAP amplitude - an indicator of variability in neural activation—assessed.

daily living in both the OL (Figure 5, left) and CL (Figure 5, right) configurations. The top and bottom plots present ECAP amplitudes and the Program \#1/Program \#2 stimulation intensity (expressed as $\mathrm{Q} / \mathrm{ph}$ ), respectively, as a function of time. For the OL case (Figure 5, left), the ECAP amplitude (average \pm S.D.) across the recording window is 15.5 $\mu \mathrm{V} \pm 10.7 \mu \mathrm{V}$. For the $\mathrm{CL}$ case (Figure 5, right), ECAP amplitudes are $9.8 \mu \mathrm{V} \pm 6.3 \mu \mathrm{V}$; by controlling stimulation intensity, ECAP variability is suppressed by $42 \%$ from 10.7 $\mu \mathrm{V}$ to $6.3 \mu \mathrm{V}$. Mean ECAP amplitudes are suppressed in the $\mathrm{CL}$ case as the system actively reduces stimulation intensity when the sensed ECAP exceeds the programmed target. Both of these effects are illustrated further in Figure 6.

The approach described above highlights the utility of ECAP variability as a quantitative measure to compare, contrast, and optimize CL SCS systems. Clinicians using these systems can first select the stimulation targets and therapy waveforms best suited for their patients, and then assess ECAP variability in both OL and CL configurations to ensure the system is providing optimal dose control across motions of daily living. Employed in this fashion, the ECAP enables direct electrophysiologic insight and automatic control for all manner of contemporary SCS therapies.

\section{Conclusion}

The past decade has been marked by substantial advances in both SCS therapies and technologies. CL SCS with the ECAP as a feedback control variable holds promise as a critical technology for providing optimized dosing of these new therapies, personalized for each patient's unique and ever-changing physiology. By providing a technological

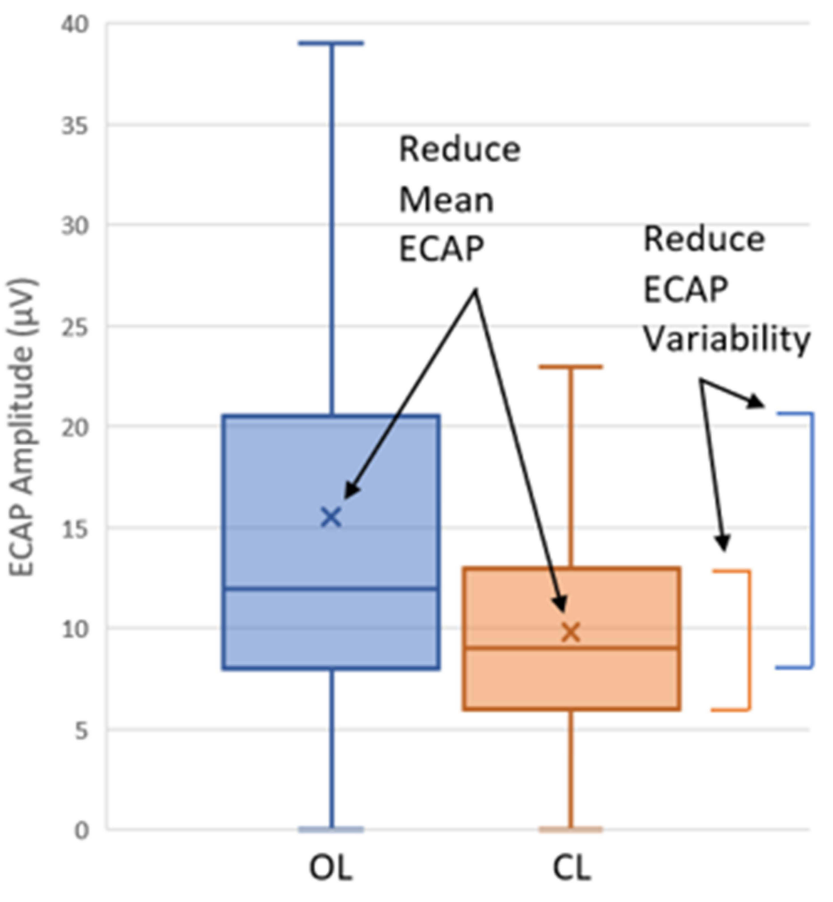

Figure 6 Evoked compound action potential (ECAP) amplitude variability suppression with closed-loop (CL, Orange) versus open-loop (OL, blue) DTM-SCS. Shown here are the mean (marked with an "X"), median, and 25th-75th percentile range for the ECAP amplitudes measured in Figure 5. The active control of stimulation charge/phase afforded by $\mathrm{CL}$ stimulation reduced variability by $42 \%$ in this example.

framework for incorporating ECAP-based control with the latest in SCS therapies, we anticipate the new direction for CL SCS elaborated in this report will define the standard of care for the SCS patients.

\section{Acknowledgments}

The authors thank Allison Foster, $\mathrm{PhD}$, an independent medical writer, who contributed to this manuscript by 
editing. We also thank Yvan Freund for his assistance with the illustrations in this manuscript.

\section{Disclosure}

Dr Ricardo Vallejo reports consultant fees from Medtronic, outside the submitted work. Dr Krishnan Chakravarthy is a consultant for and reports research funding from Medtronic, during the conduct of the study. $\mathrm{He}$ is also a consultant for Biotronik and Bioness, reports stock options from Mainstay Medical and Higgs Boson Health. $\mathrm{He}$ is the founder for NXTStim, outside the submitted work. The authors report no other conflicts of interest in this work.

\section{References}

1. Shealy CN, Mortimer JT, Reswick JB. Electrical inhibition of pain by stimulation of the dorsal columns: preliminary clinical report. Anesth Analg. 1967;46(4):489-491. doi:10.1213/00000539-19670700000025

2. Shealy CN, Mortimer JT, Hagfors NR. Dorsal column electroanalgesia. J Neurosurg. 1970;32(5):560-564. doi:10.3171/ jns.1970.32.5.0560

3. Shealy CN. Dorsal column electrohypalgesia. Headache. 1969;9 (2):99-102. doi:10.1111/j.1526-4610.1969.hed0902099.x

4. North RB, Prager JP. Chapter 45 - history of spinal cord stimulation. In: Krames ES, Peckham PH, Rezai N, Second E, editors. Neuromodulation. Academic Press; 2018:587-596. doi:10.1016/ B978-0-12-805353-9.00045-0

5. Shatin D, Mullett K, Hults G. Totally implantable spinal cord stimulation for chronic pain: design and efficacy. Pacing Clin Electrophysiol. 1986;9(4):577-583. doi:10.1111/j.1540-8159.1986. tb06614.x

6. Costandi S, Mekhail N, Azer G, et al. Longevity and utilization cost of rechargeable and non-rechargeable spinal cord stimulation implants: a comparative study. Pain Pract. 2020;20(8):937-945. doi:10.1111/papr.12926

7. Sheldon B, Staudt MD, Williams L, Harland TA, Pilitsis JG. Spinal cord stimulation programming: a crash course. Neurosurg Rev. 2020;44:709-720. doi:10.1007/s10143-020-01299-y

8. Schade CM, Schultz DM, Tamayo N, Iyer S, Panken E. Automatic adaptation of neurostimulation therapy in response to changes in patient position: results of the Posture Responsive Spinal Cord Stimulation (PRS) research study. Pain Physician. 2011;14 (5):407-417. doi:10.36076/ppj.2011/14/407

9. Schultz D, Schultz DM, Webster L, et al. Randomized trial sensor-driven position-adaptive spinal cord stimulation for chronic pain. Pain Physician. 2012;15:1-12. doi:10.36076/ppj.2012/15/1

10. Deer T, Slavin KV, Amirdelfan K, et al. Success using neuromodulation with BURST (SUNBURST) study: results from a prospective, randomized controlled trial using a novel burst waveform. Neuromodulation. 2018;21(1):56-66. doi:10.1111/ner.12698

11. Al-Kaisy A, Palmisani S, Smith TE, et al. $10 \mathrm{kHz}$ high-frequency spinal cord stimulation for chronic axial low back pain in patients with no history of spinal surgery: a preliminary, prospective, open label and proof-of-concept study. Neuromodulation. 2017;20 (1):63-70. doi:10.1111/ner.12563

12. Goudman L, De Smedt A, Eldabe S, et al. High-dose spinal cord stimulation for patients with failed back surgery syndrome: a multicenter effectiveness and prediction study. Pain. 2021;162 (2):582-590. doi:10.1097/j.pain.0000000000002035
13. Deer TR, Levy RM, Kramer J, et al. Dorsal root ganglion stimulation yielded higher treatment success rate for complex regional pain syndrome and causalgia at 3 and 12 months: a randomized comparative trial. Pain. 2017;158(4):669-681. doi:10.1097/j.pain.0000000 000000814

14. North RB, Calodney A, Bolash R, et al. Redefining spinal cord stimulation "trials": a randomized controlled trial using single-stage wireless permanent implantable devices. Neuromodulation. 2020;23 (1):96-101. doi:10.1111/ner.12970

15. Melzack R, Wall PD. Pain mechanisms: a new theory. Science. 1965;150(3699):971-979. doi:10.1126/science.150.3699.971

16. Joosten EA, Franken G. Spinal cord stimulation in chronic neuropathic pain: mechanisms of action, new locations, new paradigms. Pain. 2020;161(Suppl(1)):S104-S113. doi:10.1097/j.pain.0000000 000001854

17. Tilley DM, Lietz CB, Cedeno DL, Kelley Ca, Li L, Vallejo R. Proteomic modulation in the dorsal spinal cord following spinal cord stimulation therapy in an in vivo neuropathic pain model. Neuromodulation. 2021;24(1):22-32. doi:10.1111/ner.13103

18. Shu B, He S-Q, Guan Y. Spinal cord stimulation enhances microglial activation in the spinal cord of nerve-injured rats. Neurosci Bull. 2020;36(12):1441-1453. doi:10.1007/s12264-020-00568-6

19. Kriek N, Schreurs MWJ, Groeneweg JG, et al. Spinal cord stimulation in patients with complex regional pain syndrome: a possible target for immunomodulation? Neuromodulation. 2018;21(1):77-86. doi:10.1111/ner.12704

20. Arle JE, Mei L, Carlson KW. Fiber threshold accommodation as a mechanism of burst and high-frequency spinal cord stimulation. Neuromodulation. 2020;23(5):582-593. doi:10.1111/ner.13076

21. Vallejo R, Cedeño DL. The quest for neurobiological mechanisms of electrical stimulation of the spinal cord to reduce chronic neuropathic pain. Bioelectron Med. 2019;2(4):139-142. doi:10.2217/bem-2020-0003

22. Cedeño DL, Smith WJ, Kelley CA, Vallejo R. Spinal cord stimulation using differential target multiplexed programming modulates neural cell-specific transcriptomes in an animal model of neuropathic pain. Mol Pain. 2020;16:1744806920964360. doi:10.1177/1744806920964360

23. Smith WJ, Cedeño DL, Thomas SM, Kelley CA, Vetri F, Vallejo R. Modulation of microglial activation states by spinal cord stimulation in an animal model of neuropathic pain: comparing high rate, low rate, and differential target multiplexed programming. Mol Pain. 2021;17:1744806921999013. doi:10.1177/1744806921999013

24. Fishman MA, Calodney A, Kim P, et al. Prospective, multicenter feasibility study to evaluate differential target multiplexed spinal cord stimulation programming in subjects with chronic intractable back pain with or without leg pain. Pain Pract. 2020;20(7):761-768. doi:10.1111/papr.12908

25. Mesbah S, Angeli CA, Keynton RS, El-Baz A, Harkema SJ. A novel approach for automatic visualization and activation detection of evoked potentials induced by epidural spinal cord stimulation in individuals with spinal cord injury. PLoS One. 2017;12(10): e0185582. doi:10.1371/journal.pone. 0185582

26. Parker JL, Shariati NH, Karantonis DM. Electrically evoked compound action potential recording in peripheral nerves. Bioelectron Med. 2017;1(1):71-83. doi:10.2217/bem-2017-0005

27. DeVries L, Scheperle R, Bierer JA. Assessing the electrode-neuron interface with the electrically evoked compound action potential, electrode position, and behavioral thresholds. J Assoc Res Otolaryngol. 2016;17(3):237-252. doi:10.1007/s10162-016-0557-9

28. Parker JL, Karantonis DM, Single PS, Obradovic M, Cousins MJ. Compound action potentials recorded in the human spinal cord during neurostimulation for pain relief. Pain. 2012;153(3):593-601. doi:10.1016/j.pain.2011.11.023

29. Anaya CJ, Zander HJ, Graham RD, Sankarasubramanian V, Lempka SF. Evoked potentials recorded from the spinal cord during neurostimulation for pain: a computational modeling study. Neuromodulation. 2020;23(1):64-73. doi:10.1111/ner.12965 
30. Chakravarthy K, Bink H, Dinsmoor D. Sensing evoked compound action potentials from the spinal cord: novel preclinical and clinical considerations for the pain management researcher and clinician. J Pain Res. 2020;13:3269-3279. doi:10.2147/JPR.S289098

31. Chakravarthy K, FitzGerald J, Will A, et al. A clinical feasibility study of spinal evoked compound action potential estimation methods. Neuromodulation. 2021. doi:10.1111/ner.13510

32. Holsheimer J, den Boer JA, Struijk JJ, Rozeboom AR. MR assessment of the normal position of the spinal cord in the spinal canal. AJNR Am J Neuroradiol. 1994;15(5):951-959.

33. Chakravarthy K, Reddy R, Al-Kaisy A, Yearwood T, Grider J. A call to action toward optimizing the electrical dose received by neural targets in spinal cord stimulation therapy for neuropathic pain. $J$ Pain Res. 2021;14:2767-2776. doi:10.2147/JPR.S323372

34. Abejon D, Rueda P, Parodi E, Del Saz J. Effects of movement and postural positions in spinal cord stimulation in the new rechargeable systems. Pain Physician. 2014;17(4):345-352. doi:10.36076/ ppj.2014/17/345

35. Olin JC, Kidd DH, North RB. Postural changes in spinal cord stimulation perceptual thresholds. Neuromodulation. 1998;1 (4):171-175. doi:10.1111/j.1525-1403.1998.tb00013.x

36. Akbas M, Salem HH, Emara TH, Dinc B, Karsli B. Sensor driven-position adaptive versus conventional spinal cord stimulation in failed back surgery syndrome: a retrospective case series. Egypt J Neurol Psychiatr Neurosurg. 2019;55(1):84. doi:10.1186/s41983019-0131-6

37. Kim EK, Lee C-S, Yoo Y, et al. The long-term effectiveness of the automatic position-adaptive system in spinal cord stimulation: a retrospective comparative study with a two-year follow-up. Pain Med. 2020;21(10):2288-2297. doi:10.1093/pm/pnaa121
38. Russo M, Cousins MJ, Brooker C, et al. Effective relief of pain and associated symptoms with closed-loop spinal cord stimulation system: preliminary results of the Avalon study. Neuromodulation. 2018;21(1):38-47. doi:10.1111/ner.12684

39. Mekhail N, Levy RM, Deer TR, et al. Long-term safety and efficacy of closed-loop spinal cord stimulation to treat chronic back and leg pain (Evoke): a double-blind, randomised, controlled trial. Lancet Neurol. 2020;19(2):123-134. doi:10.1016/S1474-4422(19)30414-4

40. Kapural L, Yu C, Doust MW, et al. Novel 10-kHz high-frequency therapy (HF10 therapy) is superior to traditional low-frequency spinal cord stimulation for the treatment of chronic back and leg pain: the SENZA-RCT randomized controlled trial. Anesthesiology. 2015;123 (4):851-860. doi:10.1097/ALN.0000000000000774

41. Tai C, de Groat WC, Roppolo JR. Simulation of nerve block by high-frequency sinusoidal electrical current based on the Hodgkin-Huxley model. IEEE Trans Neural Syst Rehabil Eng. 2005;13(3):415-422. doi:10.1109/TNSRE.2005.847356

42. Bradley K. The technology: the anatomy of a spinal cord and nerve root stimulator: the lead and the power source. Pain Med. 2006;7 (suppl_1):S27-S34. doi:10.1111/j.1526-4637.2006.00120.x

43. Pilitsis JG, Chakravarthy KV, Will AJ, et al. The evoked compound action potential as a predictor for perception in chronic pain patients: tools for automatic spinal cord stimulator programming and control. Front Neurosci. 2021;15:881. doi:10.3389/fnins.2021.673998

44. Vallejo R, Chakravarthy K, Bink H, Dinsmoor D. Closed-loop, differential target multiplexed spinal cord stimulation. Abstracts from the North American Neuromodulation Society's 2021 Virtual Meeting, January 15-16, 2021. Neuromodul Technol Neural Interface. 2021;24(4):e58. doi:10.1111/ner.13385
Journal of Pain Research

\section{Publish your work in this journal}

The Journal of Pain Research is an international, peer reviewed, open access, online journal that welcomes laboratory and clinical findings in the fields of pain research and the prevention and management of pain. Original research, reviews, symposium reports, hypothesis formation and commentaries are all considered for publication. The manuscript management system is completely online and includes a very quick and fair peer-review system, which is all easy to use. Visit http:// www.dovepress.com/testimonials.php to read real quotes from published authors. 